OPEN ACCESS

Edited by:

F. Javier Moreno,

Instituto de Investigación en Ciencias

de la Alimentación (CIAL), Spain

Reviewed by:

Tadasu Urashima,

Obihiro University of Agriculture and

Veterinary Medicine, Japan

Lorena Ruiz,

Institute of Dairy Products of Asturias

(IPLA), Spain

*Correspondence:

Silvia Rudloff

silvia.rudloff@

ernaehrung.uni-giessen.de

Specialty section:

This article was submitted to

Nutrition and Food Science

Technology,

a section of the journal

Frontiers in Nutrition

Received: 23 November 2018

Accepted: 18 February 2019

Published: 12 March 2019

Citation:

Rudloff S, Kuntz S, Ostenfeldt

Rasmussen S, Roggenbuck M,

Sprenger N, Kunz C, Sangild PT and Brandt Bering S (2019) Metabolism of Milk Oligosaccharides in Preterm Pigs

Sensitive to Necrotizing Enterocolitis.

Front. Nutr. 6:23.

doi: 10.3389/fnut.2019.00023

\section{Metabolism of Milk Oligosaccharides in Preterm Pigs Sensitive to Necrotizing Enterocolitis}

\author{
Silvia Rudloff ${ }^{1,2 *}$, Sabine Kuntz ${ }^{1}$, Stine Ostenfeldt Rasmussen ${ }^{3}$, Michael Roggenbuck ${ }^{4}$, \\ Norbert Sprenger ${ }^{5}$, Clemens Kunz ${ }^{1}$, Per Torp Sangild ${ }^{3}$ and Stine Brandt Bering ${ }^{3}$ \\ ${ }^{1}$ Institute of Nutritional Sciences, Justus Liebig University Giessen, Giessen, Germany, ${ }^{2}$ Department of Pediatrics, Justus \\ Liebig University Giessen, Giessen, Germany, ${ }^{3}$ Section for Comparative Pediatrics and Nutrition, University of Copenhagen, \\ Frederiksberg, Denmark, ${ }^{4}$ Section of Microbiology, Department of Biology, University of Copenhagen, Copenhagen, \\ Denmark, ${ }^{5}$ Nestlé Research Centre, Nestec S.A., Lausanne, Switzerland
}

Human milk oligosaccharides (HMO) are major components of breast milk that may have local effects in the gastrointestinal tract and systemic functions after being absorbed, both depending on their metabolism. Using preterm pigs, we investigated the metabolic fate of $\mathrm{HMO}$ in three experiments with two different $\mathrm{HMO}$ blends. In addition, we examined effects on the colonic microbiota in the presence or absence of necrotizing enterocolitis (NEC). Thus, preterm pigs $(n=112)$ were fed formula without or with HMO supplementation (5-10) $\mathrm{g} / \mathrm{L}$ of a mixture of 4 (4-HMO) or $>25 \mathrm{HMO}(25-\mathrm{HMO})$ for 5 (Experiment 1 and 2) or 11 days (Experiment 3). Individual $\mathrm{HMO}$ were quantified in colon contents and urine using MALDI-TOF-MS (matrix-assisted laser desorption ionization mass spectrometry) and HPAEC-PAD (high-performance anion-exchange chromatography with pulsed amperometric detection). Microbial colonization was analyzed by sequencing of $16 \mathrm{~S}$ rRNA gene tags. Intestinal permeability was measured by lactulose to mannitol ratio in urine. HMO supplemented to formula were detected in urine and colon contents in preterm piglets after 5 and 11 days in all three experiments. The amount of $\mathrm{HMO}$ excreted via the gut or the kidneys showed large individual variations. Microbial diversity in the colon changed from high levels of Firmicutes (dominated by Clostridium) at day 5 (Exp 2) to high levels of Proteobacteria dominated by Helicobacter and Campylobacter at day 11 (Exp 3). Colonic microbiota composition as well as HMO excretion pattern varied greatly among piglets. Interestingly, the 5-day supplementation of the complex 25-HMO blend led to low concentrations of 3-fucosyllactose (FL) and lacto-N-fucopentaose (LNFP) I in colonic contents, indicating a preferred utilization of these two HMO. Although the interpretation of the data from our piglet study is difficult due to the large individual variation, the presence of Bifidobacteria, although low in total numbers, was correlated with total $\mathrm{HMO}$ contents, and specifically with $2^{\prime} \mathrm{FL}$ levels in colonic content. However, early supplementation of formula with $\mathrm{HMO}$ did not affect NEC incidence.

Keywords: human milk oligosaccharides (HMO), preterm pigs, metabolism, necrotizing enterocolitis (NEC), microbiota, formula 


\section{INTRODUCTION}

Increasing evidence supports the hypothesis that benefits of human milk for infants are partly explained by the abundance of complex oligosaccharides. Proposed functions concern their interactions with gut microbiota, the prevention of pathogen adhesion to the epithelium, effects on gut maturation or an influence on the developing brain (1-7). So far, the first human trials with one or two single human milk oligosaccharides (HMO), 2'fucosyllactose (FL) or 2'FL plus lacto-N-neo-tetraose (LNnT), in term infants demonstrated that the new formula were safe and lead to growth rates comparable to those found in term human milk-fed infants $(8,9)$. Within this context, metabolic aspects of HMO are an important issue as, for example, currently discussed systemic effects such as an influence on inflammatory processes or on brain functions and activity require the preceding absorption of HMO. Indeed, HMO have been detected in the circulation of breast-fed infants $(10,11)$. To investigate metabolic pathways of single HMOs, studies in infants are limited due to the low HMO availability and also for ethical concerns. Therefore, the selection of an appropriate alternative animal model is an important point to consider. In animals, it has been shown that some HMO can be absorbed $(12,13)$. As rats, however, do not seem to be suitable for HMO metabolic studies, we used pigs which may have a translational advantage based on the physiological similarity between pigs and humans with regard to the gastrointestinal tract, its delayed maturation and high natural sensitivity to necrotizing enterocolitis (NEC) after preterm birth $(14,15)$.

In preterm infants, NEC is a major cause of morbidity and mortality affecting $5-10 \%$ of infants $<1,500 \mathrm{~g}$ with a mortality of $20-30 \%(16,17)$. Breast-fed infants were shown to have lower NEC incidences than formula-fed infants (18). In rat pups, three studies showed that the addition of HMO such as disialyl-lacto-N-tetraose (DSLNT), $2^{\prime} \mathrm{FL}$ or $2^{\prime} \mathrm{FL}$ plus sialylated galactooligosaccharides, the latter not being present in human milk, decreased NEC incidence (19-21). A potential mechanism for the observed effects may be the upregulation of mucins, and concomitant decrease in intestinal permeability which has recently been shown by pooled HMO (22).

We hypothesized that HMO supplementation of formula leads to fecal and/or urinary excretion of intact HMO at various amounts depending on the prevailing microbiota. If so, not only gastrointestinal but also systemic functions are to be expected. In addition, we aimed at investigating whether HMO supplemented formula affect bacterial colonization and thereby improve NEC resistance in preterm pigs. Because of the potential for synergistic effects among different HMO, we investigated for the first time effects of a large range of HMO, e.g., mixtures containing either 4 or $>25 \mathrm{HMO}$, the latter reflecting the complex oligosaccharide composition in human milk. Clinical and physiological effects on the gastrointestinal tract have been reported previously (23). Here, we focus on HMO metabolism and its potential relations to bacterial gut colonization.

\section{MATERIALS AND METHODS}

Information on animal housing is given in the preceeding paper (19). The HMO blends were provided by Glycom A/S (Lyngby, Denmark). The study was approved by the Danish National Committee on Animal Experimentation (license number 201215-2934-00193).

\section{Experimental Design and Sample Collection}

Experiment 1,2, and 3 were carried out in preterm born pigs (delivered by Cesarean section at $\sim 90 \%$ of gestation) using different blends of $\mathrm{HMO}$ and varying length of exposure time (5 or 11 days postpartum) as described previously (Figure 1) (23). HMO blends were chosen to either represent the most abundant individual structures in human milk (4-HMO) or to additionally cover more closely the complex range of oligosaccharides known for human milk with more than 25 compounds present (25HMO) (Table 1).

Preterm pigs $(n=112)$ in each of the three experiments were divided into two groups (control and HMO-treated) based on birth weight and gender (Figure 1) The macronutrient content of the diets is described in Table 2.

Dietary treatments as well as laboratory analyses were blinded to investigators and all animal procedures (23). In Experiment 1 and 2, preterm pigs were fed standard formula with or without HMO for 5 days. In Experiment $1(n=44), 5 \mathrm{~g} / \mathrm{L} 4-\mathrm{HMO}$ or maltodextrin (controls) were added to the formula, whereas in Experiment $2(n=38), 7 \mathrm{~g} / \mathrm{L} 25-\mathrm{HMO}$ were supplemented to account for the lower abundance of the major HMO used in the 4-HMO blend (23); in Experiment 3 (11 days, $n=30$ ), preterm pigs were fed with 10 and $5 \mathrm{~g} / \mathrm{L} 4$ - $\mathrm{HMO}$ for the first 4 days and up to 11 days, respectively; the control group received ready-to-feed infant formula (RTF-IF, controls) without HMO supplementation. The infant formula was chosen to include a more translational aspect into the longer term experiment. At the end of the experiments, pigs were anesthetized (Zoletil 50, zolazepam/tiletamin; Boehringer Ingelheim, Copenhagen, Denmark), euthanized by injection with sodium pentobarbital, and biological material was collected. Colonic content and urine taken from the bladder were snap-frozen for determination of HMO, colonic microbiota composition, and intestinal permeability. Tissue samples were collected for evaluation of NEC as reported previously (19). Collection of colonic contents and urine could not be achieved from all animals in amounts necessary for all analyses; thus, the number of samples analyzed is given in the corresponding figures.

\section{Evaluation of NEC and Intestinal Permeability}

Stomach, small intestine (proximal, middle, and distal regions), and colon were evaluated for NEC lesions and given a score from 1 (absence of macroscopic lesions), 2 (local hyperemia), 3 (hyperemia, milch hemorrhage, extensive edema), 4 (extensive hemorrhage), 5 (local necrosis, and pneumatosis intestinalis) to 6 (extensive necrosis and intramural gas cysts). NEC was 


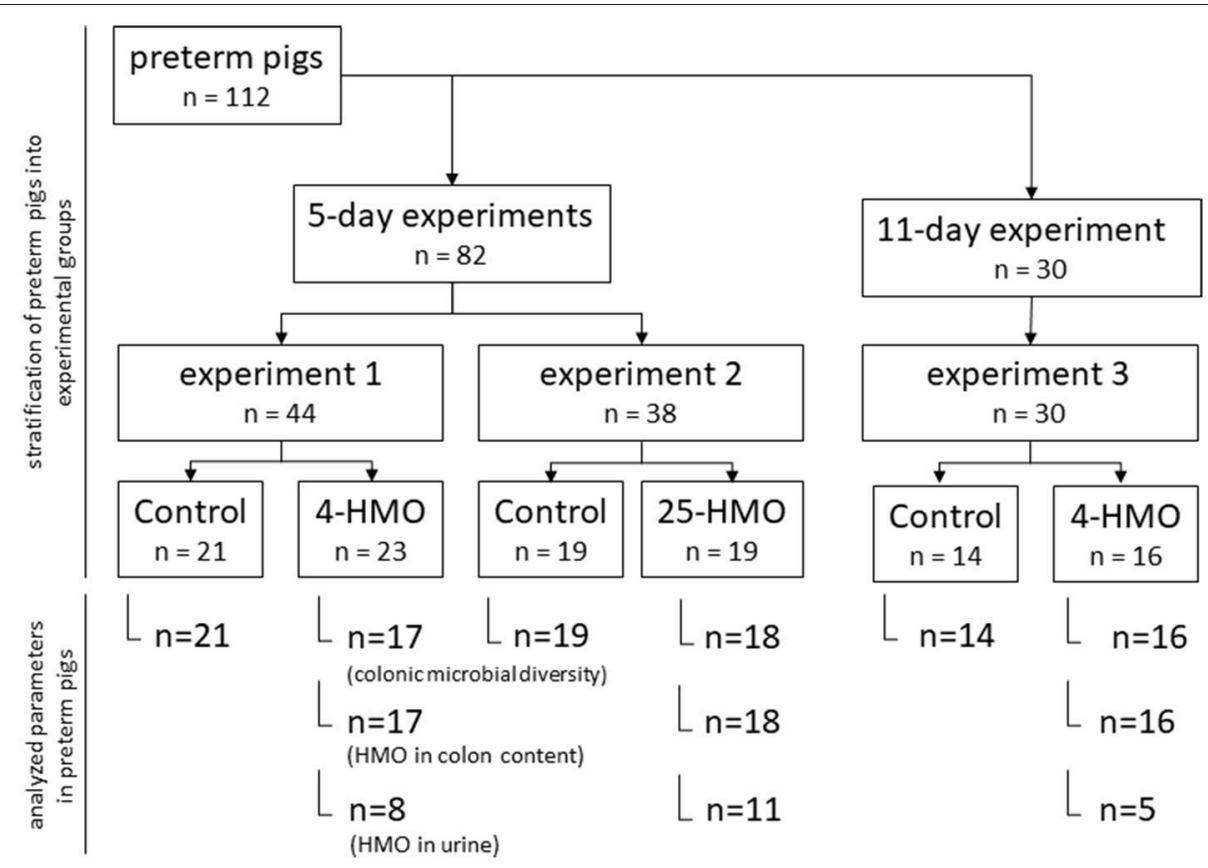

FIGURE 1 | Experimental design for 5-day and 11-day experiments in preterm pigs.

defined as a score of $\geq 3$ in any of the evaluated regions (23). Intestinal permeability was determined by the lactulose to mannitol ratio in urine. Pigs were fed 5\% lactulose and 5\% mannitol (15 mL/kg body weight) $3 \mathrm{~h}$ prior to euthanasia, and were fed half a bolus of their respective diets $1.5 \mathrm{~h}$ prior to euthanasia. Urine was collected at euthanasia, and lactulose and mannitol concentrations were analyzed spectrophotometrically as described previously (24).

\section{Analytical Procedures for HMO Quantification}

High-performance anion-exchange chromatography with pulsed amperometric detection (HPAEC-PAD) was applied for discrimination of stereoisomeric HMO, e.g., lacto-N-tetraose (LNT) and LNnT as well as for identification and quantification of HMO in urine and colonic contents using external standard oligosaccharides (Carbosynth Ltd, Berkshire, UK; Dextra, Reading, UK; Elicityl, Crolles, France) $(25,26)$. To verify the presence of HMO determined by HPAEC-PAD, matrix-assisted laser desorption ionization mass spectrometry (MALDI-TOFMS) was used as described (26). Briefly, samples (urine and solubilized colon contents) were centrifuged after the addition of pure water. For normalization of the amount of urinary constituents applied to the extraction procedure, creatinine concentration was determined colorimetrically (R\&D Systems, Heidelberg, Germany). Solid phase extraction with porous graphitic carbon cartridges (for colonic contents: HyperSep96 Wells, $25 \mathrm{mg}$; for urine: HyperSep Hypercarb $50 \mathrm{mg}$; Thermo Scientific, Bellefonte PA, USA) was performed via a Hamilton Microlab Starlet liquid handling system (Hamilton Robotics, Reno, NV, USA) or manually for urine samples. The conditions for cartridge equilibration as well as the elution of oligosaccharides have been described previously $(25,26)$.

After solid phase extraction, oligosaccharides were dried overnight in a vacuum centrifuge and resuspended in water. An HPAEC-PAD system (Dionex ICS-5000) equipped with a CarboPac PA-1 and a guard column was operated using the Chromeleon 6.80 software (ThermoFisher Scientific, Dreieich, Germany). The running parameters at a constant flow rate of 0.5 $\mathrm{mL} / \mathrm{min}$ were as follows: $0.1 \mathrm{~mol} / \mathrm{L}$ sodium hydroxide from 0 to $15 \mathrm{~min}$, followed by a linear gradient up to $0.25 \mathrm{~mol} / \mathrm{L}$ sodium acetate in $0.1 \mathrm{~mol} / \mathrm{L}$ sodium hydroxide for $87 \mathrm{~min}$. External oligosaccharide standards were used for peak identification and the area under the curves were determined. Individual 4-point calibrations were used for quantification of oligosaccharides in extracts from urine and colonic contents. Mass spectra from the same samples in triplicate determinations were acquired using an Ultraflex I instrument (Bruker Daltonics, Bremen, Germany). Oligosaccharide profiles were acquired in positive-ion mode over a mass range of $\mathrm{m} / \mathrm{z} 340-3,200$. Data acquisition and analysis were performed by flexControl and flexAnalysis 3.0 software (Bruker Daltonics, Bremen, Germany), respectively $(25,26)$.

\section{Identification and Characterization of Microorganisms}

Colonic bacterial microbiota composition was determined by tag-encoded $16 \mathrm{~S}$ rRNA gene MiSeq-based high throughput sequencing (Illumina, San Diego, CA, USA) as published recently (27). Briefly, DNA was extracted from $0.5 \mathrm{~g}$ colon content using the PowerSoil DNA Isolation Kit (MoBio Laboratories). The V3$\mathrm{V} 4$ region of the $16 \mathrm{~S}$ rRNA gene was amplified with the universal 
TABLE 1 | HMO compositions in 4-HMO and 25-HMO blends.

\begin{tabular}{|c|c|c|c|c|}
\hline No. & Compound ${ }^{a}$ & Abbreviation & $\begin{array}{l}\text { Amount in 4-HMO } \\
(\mathrm{g} / 100 \mathrm{~g} \text { blend })\end{array}$ & $\begin{array}{c}\text { Amount in } 25-\mathrm{HMO} \\
\text { (g/100 } \mathrm{g} \text { blend })\end{array}$ \\
\hline 1 & 2'Fucosyllactose & $2^{\prime} \mathrm{FL}$ & 61.9 & 18.7 \\
\hline 2 & Lacto-N-neo-tetraose & LNnT & 10.3 & 7.9 \\
\hline 3 & Lacto-N-tetraose & LNT & 14.4 & 7.9 \\
\hline 4 & 6'Sialyllactose & $6^{\prime} S L$ & 10.3 & 3.6 \\
\hline 5 & 3-Fucosyllactose & 3-FL & - & 12.1 \\
\hline 6 & Difucosyllactose & DF-L & - & 2.7 \\
\hline 7 & Lacto-N-fucopentaose I & LNFP I & - & 6.5 \\
\hline 8 & Lacto-N-fucopentaose II & LNFP ॥ & - & 2.7 \\
\hline 9 & Lacto-N-fucopentaose III & LNFP III & - & 1.8 \\
\hline 10 & Sialyllacto-N-tetraose a & LST a & - & 0.9 \\
\hline 11 & Sialyllacto- $\mathrm{N}$-neo-tetraose c & LST c & - & 2.7 \\
\hline 12 & 3'Sialyllactose & 3'SL & - & 3.7 \\
\hline 13 & Disialyllacto-N-tetraose & DSLNT & - & 7.0 \\
\hline \multirow[t]{3}{*}{$14-32$} & Other oligosaccharides ${ }^{b}$ & - & 3.1 & 8.6 \\
\hline & Others (mainly salt) & & - & 2.9 \\
\hline & TOTAL & & 100.0 & 100.0 \\
\hline
\end{tabular}

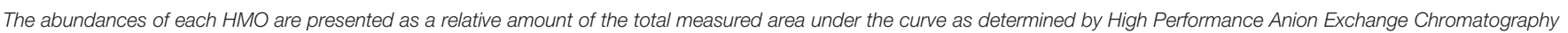
with Pulsed Amperometric Detection (HPAEC-PAD) for each HMO.

a Compounds 1-13 (90\% of all oligosaccharides in the 25-HMO blend).

${ }^{b}$ Compounds 14-32 (not quantified).

TABLE 2 | Composition of the formula used in Experiment 1-3.

\begin{tabular}{lcc}
\hline & Standard Formula $^{\mathbf{a}}$ & RTF-IF $^{\mathbf{b}}$ \\
\hline Energy, KJ/L & 4,115 & 3,400 \\
Protein, g/L & 73 & 29 \\
Fat, g/L & 59 & 40 \\
Carbohydrates, g/L & 42 & 84 \\
Lactose, g/L & - & 36 \\
Maltodextrin, g/L & 46 & 45 \\
Oligosaccharides, g/L & $-\mathrm{c}$ & $-\mathrm{d}$ \\
\hline
\end{tabular}

a Piglet formula mixed from Pepdite 2-0, Liquigen-MCT (SHS International, Liverpool, UK), and Lacprodan DI-9224 (Arla Food Ingredients, Aarhus, Denmark).

${ }^{b}$ RTF-IF, ready-to-feed infant formula for preterm infants (Alprem, Clinic 1, Nestlé Nutrition S.A., Barcelona, Spain).

c Supplemented with maltodextrin (control) or HMO [Experiment 1 (5-day): $5 \mathrm{~g} / \mathrm{L} 4$ 4-HMO; Experiment 2 (5-day): $7 \mathrm{~g} / \mathrm{L}$ 25-HMO].

${ }^{d}$ With and without HMO supplementation [Experiment 3 (11-day): $10 \mathrm{~g} / \mathrm{L} \mathrm{4-HMO}$ blend during the first 4 days, then $5 \mathrm{~g} / \mathrm{L}$ as in Experiment 1].

prokaryotic primers 515F (5'-GTGCCAGCMGCCGCGGTAA$\left.3^{\prime}\right)$ and 806R (5'-GGACTACHVGGGTWTCTAAT-3' in a first PCR revealing amplicon lengths of 290 bp (28-30). In a second PCR, adapters compatible with the Nextera Index Kit (Illumina) where attached to the amplicons. After the amplification of fragments with adapters and tags, these were purified and clean constructs were quantified prior to library pooling, by using a Qubit fluorometer (Invitrogen, Carlsbad, CA, USA). The $2 \times 250$-bp sequencing reaction followed the standard procedure of Illumina MiSeq for pair-end reads. After sequence generation, the reads were demultiplexed and paired followed by a clean-up step to truncate primers, remove low quality sequences, and chimeras following the default settings of the UPARSE pipeline. Operational taxonomic units (OTUs) were picked with USEARCH at $>97 \%$ sequences identity and classified using Mothur (v.1.33.3) and the RDP database (31, 32). Uneven sequencing depth was corrected using a zero-inflated Gaussian distribution implanted in the R package of MetagenomeSeq (33).

\section{Statistical Analysis}

Statistical analyses were carried out using GraphPad Prism 6.0.1.3 (GraphPad Software); results were expressed as medians with IQR (interquartile range, 10th-90th percentiles). D'Agostino Pearson omnibus normality tests were used to determine whether data sets were well-modeled by normal distribution. If necessary, $\log$ transformation was used. As indicated, data were analyzed by ANOVA or student $t$-test. Corrections for multiple comparisons were made using the Holm-Sidak method. Differences were considered significant at $P<0.05$.

Associations were described by using Spearman's or Pearson correlation coefficients. Correlation analyses were shown as correlation coefficient $r$ with $95 \%$ confidence interval.

Taxonomic relative abundance data (OTUs $>5 \%$ ) were used to calculate correlations between HMO consumption and fecal bacteria abundance. A Spearman's correlation was used to describe associations. Differences between groups were assessed using ANOVA and Tukey multiple-comparison test.

\section{RESULTS}

\section{Identification and Characterization of HMO}

To analyze oligosaccharides in the HMO blends as well as in urine and colonic contents, we applied HPAEC-PAD and MALDITOF-MS. In the complex 25-HMO blend (Table 1), 13 HMO 
were quantified, which, however, comprised almost $90 \%$ of all HMO present (Figure 2A). In Figure 2B, exemplary MALDITOF-MS spectra of urine from pigs fed a control formula or a formula supplemented with 4-HMO are shown. The control pig received a standard formula containing higher saccharides (maltodextrin), which was reflected by hexose oligomers of different lengths. For the pig receiving HMO, the mass-to charge ratio $(\mathrm{m} / \mathrm{z})$ of 511,656 , and 730 represent $2^{\prime} \mathrm{FL}, 6^{\prime} \mathrm{SL}$, and the isomers LNT and LNnT deriving from the 4-HMO blend.

\section{HMO in Colon Contents and Urine}

Five-Day Supplementation of 4-HMO (Experiment 1) or of 25-HMO (Experiment 2)

The total colonic HMO content was 46.9 (20.6-337.7) $\mathrm{mg} / \mathrm{g}$ dry weight and in urine $16.2(3.3-48.8) \mathrm{mg} / \mu \mathrm{mol}$ creatinine
(Figure 3A). Large variations were observed in both the total $\mathrm{HMO}$ concentrations and individual components of the 4-HMO blend. There seemed to be no specificity in the HMO degradation or absorption. The 25-HMO blend, designed to be closer to that normally found in human milk than the 4-HMO was used in Experiment 2. With the 25-HMO blend, the total colonic HMO content was $9.8(0-29.1) \mathrm{mg} / \mathrm{g}$ dry weight and in urine $56.6(9.3-127.3) \mathrm{mg} / \mu \mathrm{mol}$ creatinine (Figure 3B). As with the 4$\mathrm{HMO}$ blend, supplementation with the 25-HMO blend revealed a large individual variability in HMO excretion via colon contents and urine. In contrast to $2^{\prime} \mathrm{FL}$ in both $\mathrm{HMO}$ blends, 3-FL was metabolized differently. Whereas, the colon contents of $2^{\prime} \mathrm{FL}$ varied greatly [0.9 (0-6.3) $\mathrm{mg} / \mathrm{g}$ dry weight], the variation of the 3-FL excretion was small with only low amounts detectable in the colon and urine $[0.01(0-0.9) \mathrm{mg} / \mathrm{g}$ dry weight and $0.5(0-1.1)$

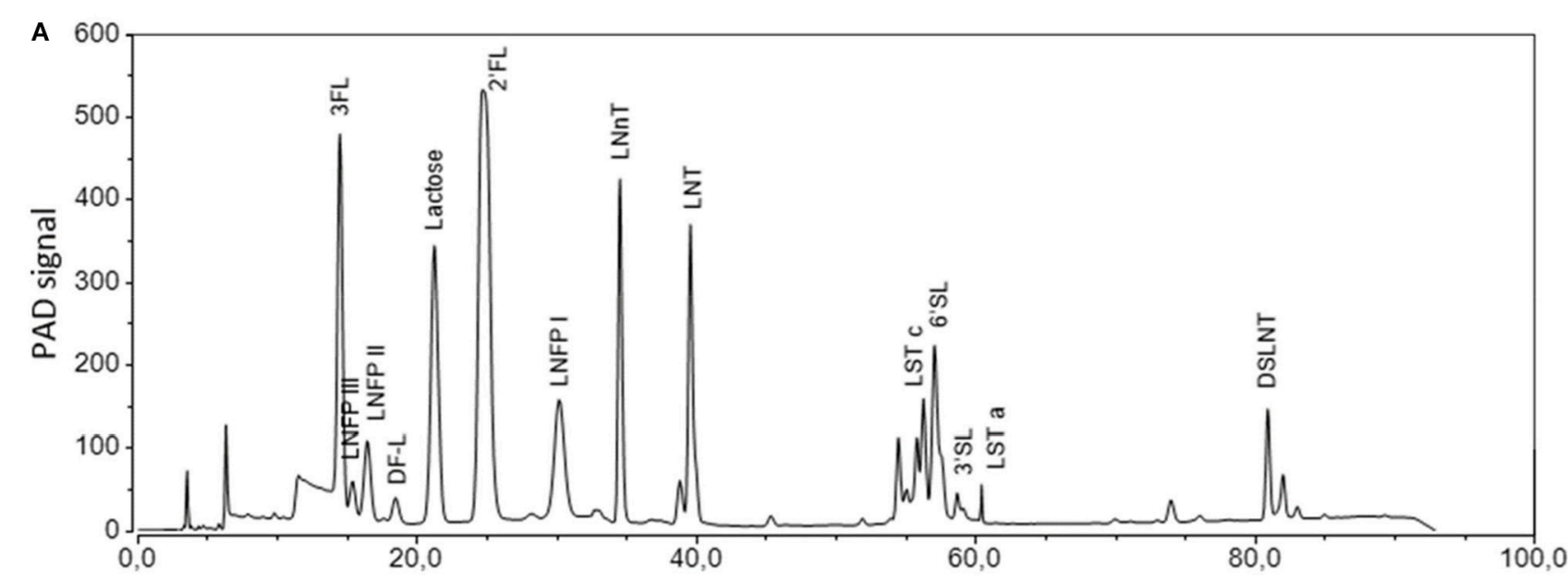

B
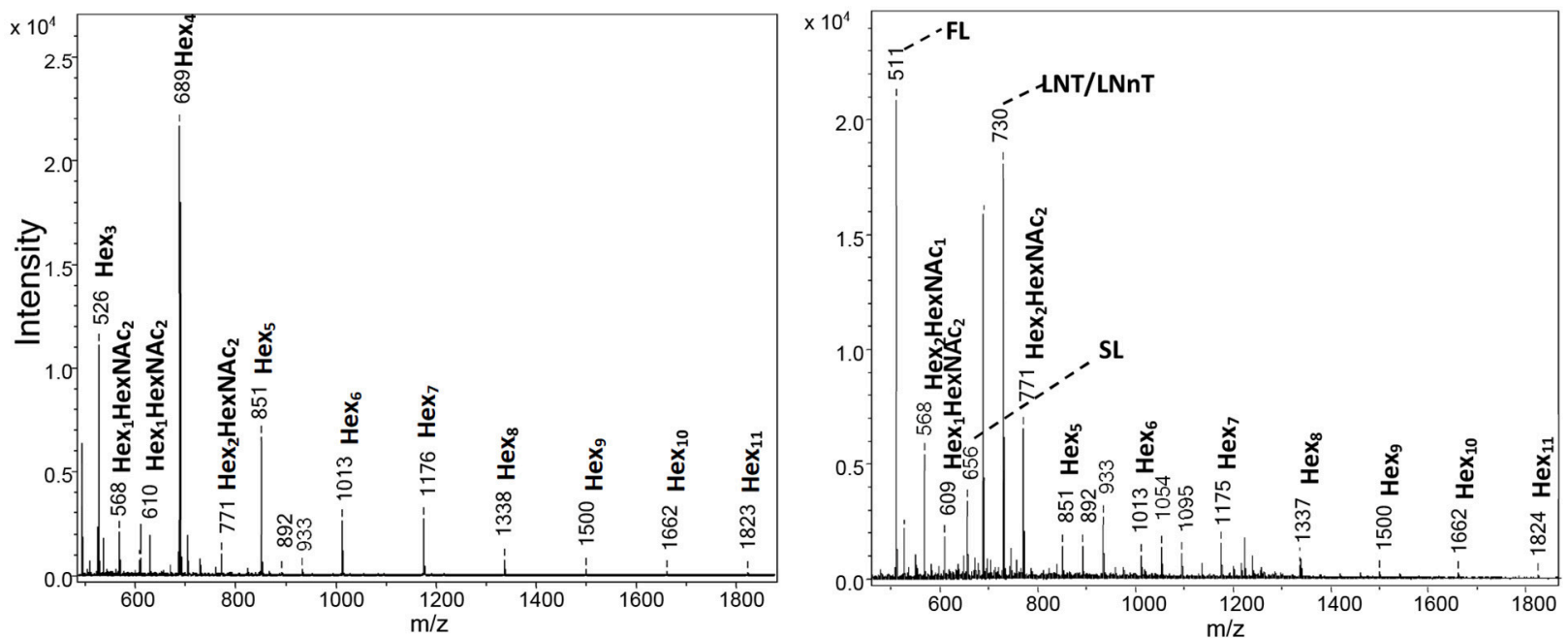

FIGURE 2 | Analytical procedures comprising (A) high-performance anion-exchange chromatography with pulsed amperometric detection (HPAEC-PAD). From the complex HMO blend named 25-HMO, 13 compounds were used for quantification (comprise $90 \%$ of all oligosaccharides in this blend), and (B) matrix-assisted laser desorption ionization mass spectrometry (MALDI-TOF-MS). The panels show a representative profile of urine from piglets from Experiment 1 fed the standard formula without (left panel) and with (right panel) a 4-HMO blend. The mass-to-charge $(\mathrm{m} / \mathrm{z})$ values are given as nominal mass for [M+Na] ${ }^{+}$ions. 
$\mathrm{mg} / \mu \mathrm{mol}$ creatinine, respectively], indicating an almost complete fermentation. LNFP I, one of the quantitatively major HMO in the 25-HMO blend, also showed very low concentrations in colon content $[0.01(0-0.09) \mathrm{mg} / \mathrm{g}$ dry weight]. Its structural isomers, LNFP II and III, were present in much lower concentrations in the 25-HMO blend, but were detectable in higher amounts in colon contents than LNFP I. Compared to the low amount of 3FL in both colonic content and urine, LNFP I was low in colonic content but showed a large individual variation in urine.

\section{Eleven-Day Supplementation of the 4-HMO Blend (Experiment 3)}

In this 11 days lasting experiment, the HMO content in the colon as well as in urine varied largely with an average of 13.8 (0160.6) $\mathrm{mg} / \mathrm{g}$ dry weight and $21.4(5.8-97.0) \mathrm{mg} / \mu \mathrm{mol}$ creatinine, respectively (Figure $3 \mathrm{C}$ ). Similar to the 5-day experiment, there seemed to be no specificity in HMO metabolism as the same proportions between the $4 \mathrm{HMO}$ were found in colonic content and urine after 11 days, however, some pigs showed higher

\section{A}

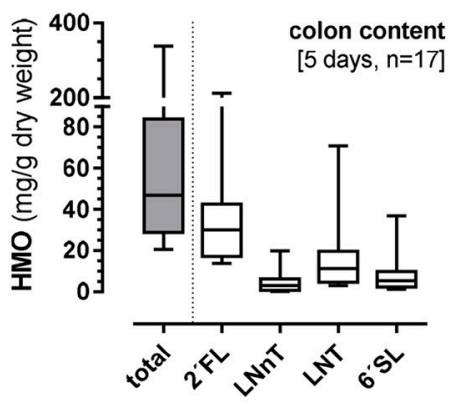

B

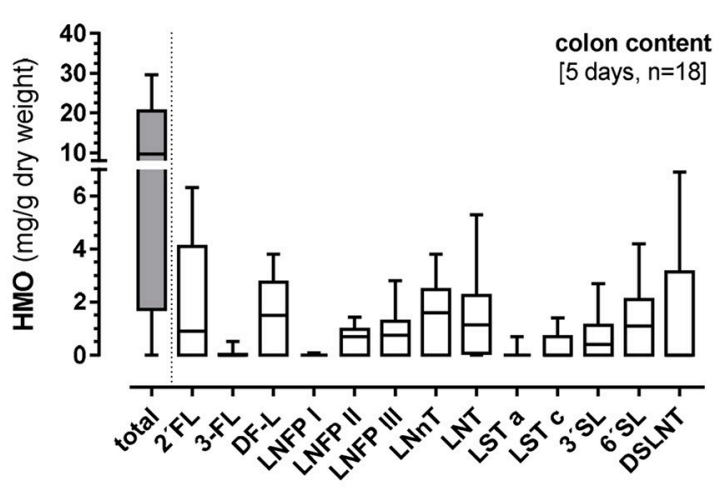

C

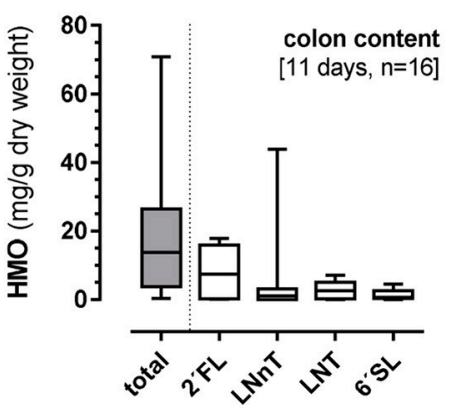

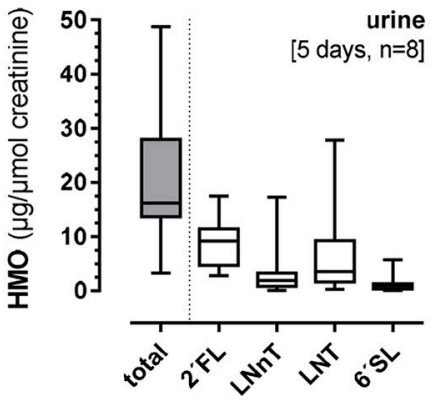
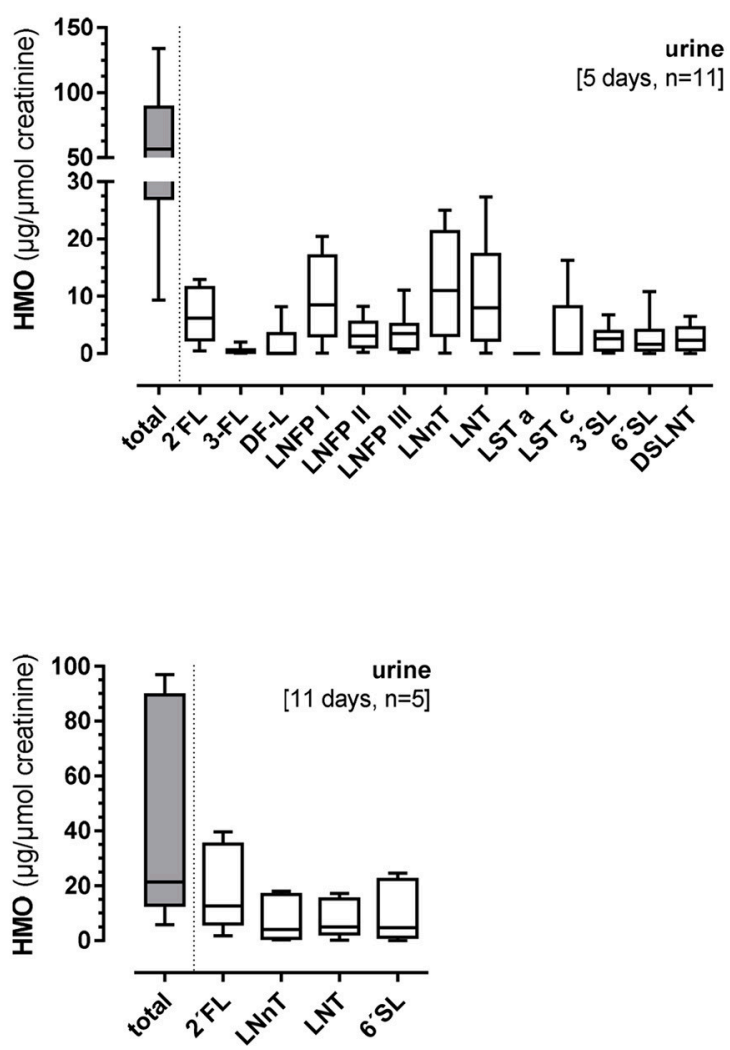

FIGURE 3 | Total and individual concentrations of human milk oligosaccharides (HMO) in colon contents (mg/g dry weight) and urine (mg/ $\mathrm{mmol}$ creatinine) after (A) 5-day supplementation of 4-HMO or (B) 5-day supplementation of 25-HMO, and (C) after 11-day feeding of 4-HMO. Data are presented as box plots with median and interquartile range (IQR, 10-90th percentiles). 
utilization than others. For example, if the concentration of $2^{\prime} \mathrm{FL}$ was high in colon content and urine, then LNnT, LNT, and 6'SL excretion was high in the same animal (Figure 4).

\section{Colonic Microbial Compositions and HMO Concentrations}

Figure 5 shows the most prevailing bacterial genera. At the phylum level, only Firmicutes were detected in the 5-day experiment when preterm piglets were fed the 4-HMO blend, with Clostridium, Enterococcus, and Lactobacillus being most abundant (Figure 5A). When the complex 25-HMO blend was supplemented within a 5-day period, only few more genera were present. Again, Clostridium and Enterococcus accounted for more than half of all bacteria (Figure 5B).

The 11-day supplementation of the 4-HMO blend revealed an increasingly complex microbial colonization with Bacteroidetes, Actinobacteria, Proteobacteria, Firmicutes, and Fusobacteria (Figure 5C). In contrast to the 5-day experiments, Proteobacteria was the most abundant phylum with mainly Camphylobacter and Helicobacter at the genera level. Furthermore, at the genus level, some Bifidobacteria which are well-known for their abilities to consume HMO, were detected in both HMO-fed and control pigs, with only slightly higher proportions in $\mathrm{HMO}$-fed piglets compared to controls $(P<0.11)$. Nevertheless, an inverse correlation was found for Bifidobacteria density and total HMO concentrations in colon contents $(P<0.05)$. An even stronger inverse correlation was found for $2^{\prime} \mathrm{FL}$, but not for other HMO (Figure 6). No correlation was found for Lactobacillus and Bacteroides.

\section{Correlation of HMO and Cumulative NEC Score and Gut Permeability}

Data for clinical outcomes and NEC scores for all 112 pigs have been described previously (23). When comparing HMO levels in the colonic content with the cumulative NEC score, and HMO levels in urine with intestinal permeability, a significant correlation ( $r=-0.764 ; P=0.0033$ ) was found for HMO in colon content of pigs with NEC in the 5-day experiment receiving the 25-HMO blend. This inverse association was also observed at the individual HMO level for various single oligosaccharides. However, there was no significant correlation between HMO levels in urine and intestinal permeability (Table 3).

\section{DISCUSSION}

We investigated effects of infant formula supplemented with complex HMO mixtures in a preterm pig model. We documented the metabolism of HMO from blends that either contained the
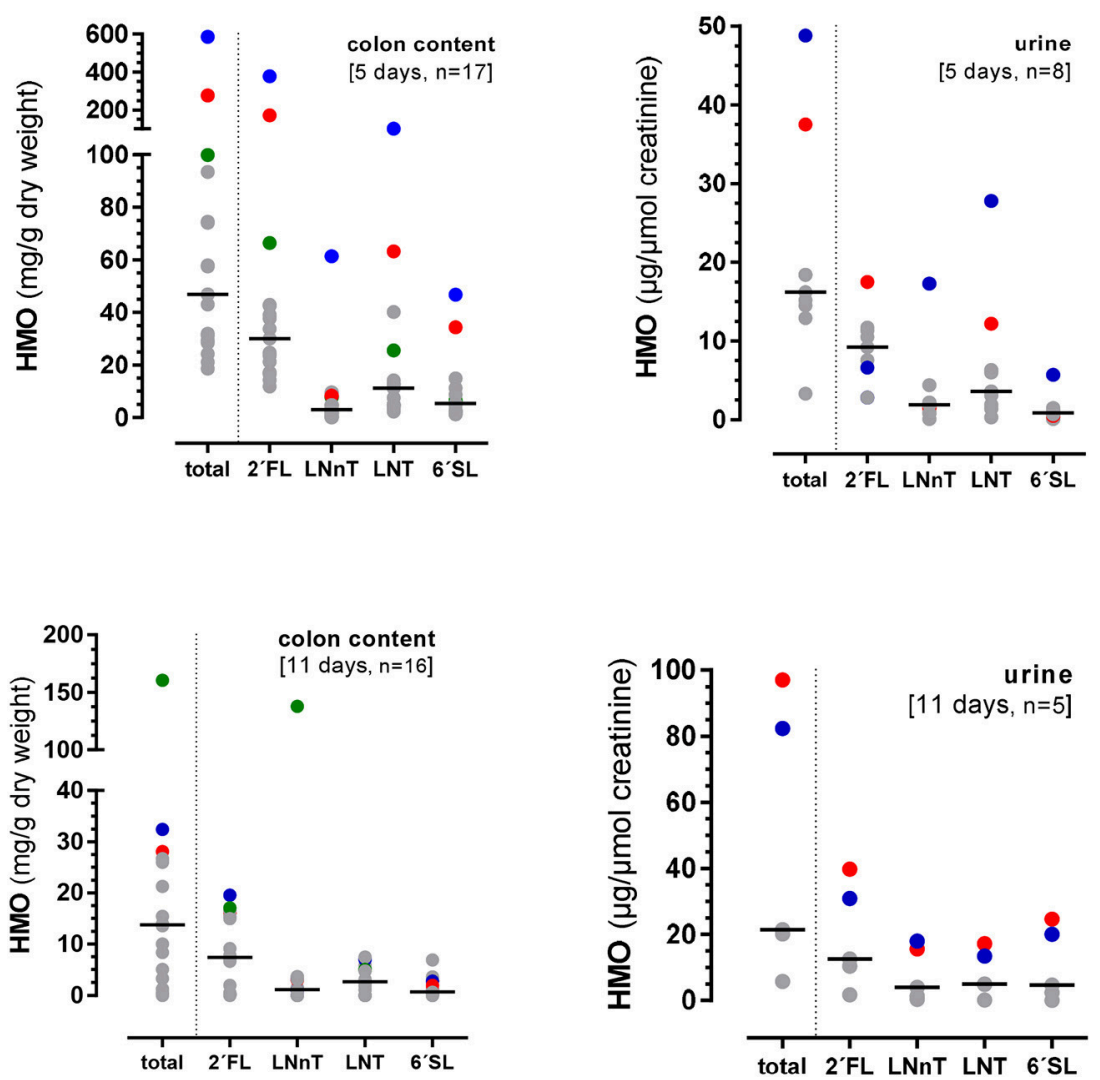

FIGURE 4 | Excretion of total HMOs and individual HMOs from the 4-HMO blend containing 2'FL, LNnT, LNT, and 6'FL via the colon and urine. Different color dots represent $\mathrm{HMO}$ concentrations from different pigs with higher $\mathrm{HMO}$ excretions. 


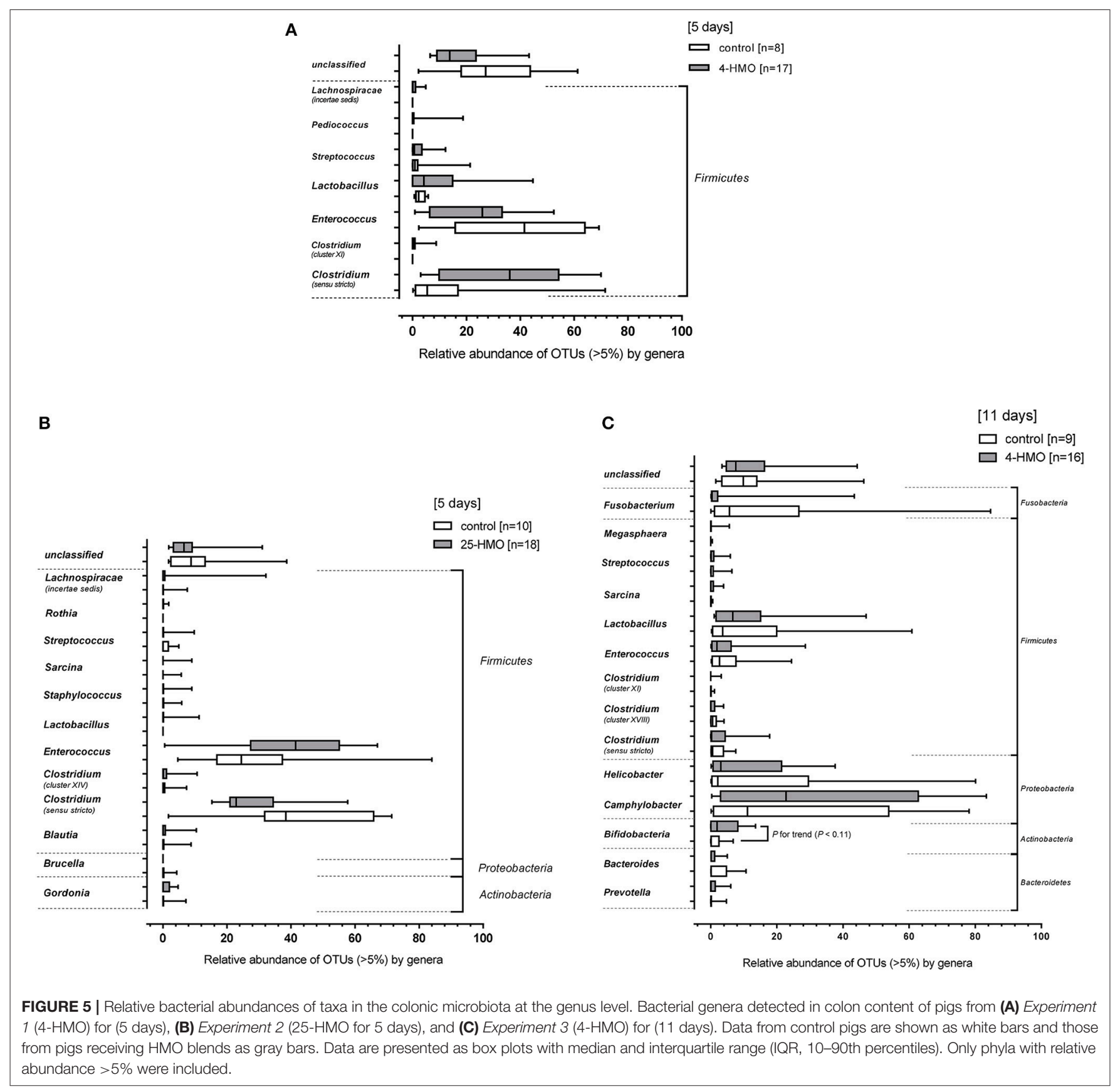

major HMO (4-HMO blend) or the most abundant HMO (25HMO blend) at a ratio of neutral and acidic oligosaccharides of about 80:20 which has been reported for human milk (1). Analyzing urine and feces across three different experiments, we found large individual variations in HMO excretion. This was particularly the case in the 4-days experiment (Figure 4) whereas after 11 days this large variation was markedly reduced. Due to the low number of individual samples, it is unclear at the moment whether the excretion is related to the relative distribution of $\mathrm{HMO}$ in the formula diet.

For 3-FL and LNFP I, there seems to be a structure-specific metabolism of this single HMO. Their concentration in the colon was very low although they belonged to the major HMO components in the 25-HMO blend. LNFP I is typically found in human milk of secretors (about $70-80 \%$ of the population) and shares similar functional epitopes with $2^{\prime} \mathrm{FL}$ which is currently considered to be a highly potent HMO. 3-FL regularly occurs in human milk, but has until now received limited attention as compared to its structural isomer $2^{\prime} \mathrm{FL}(25)$. In addition, the linkage of fucose on $\mathrm{C}$-atom 2 of the galactose moiety of $2^{\prime} \mathrm{FL}$ turns it into a structure with a high potential as an anti-adhesive and anti-inflammatory component. Although the secretor-specific $\alpha 1-2$-fucose linkage is missing in 3-FL, an in vitro inhibiton of Norovirus binding has been reported for both 

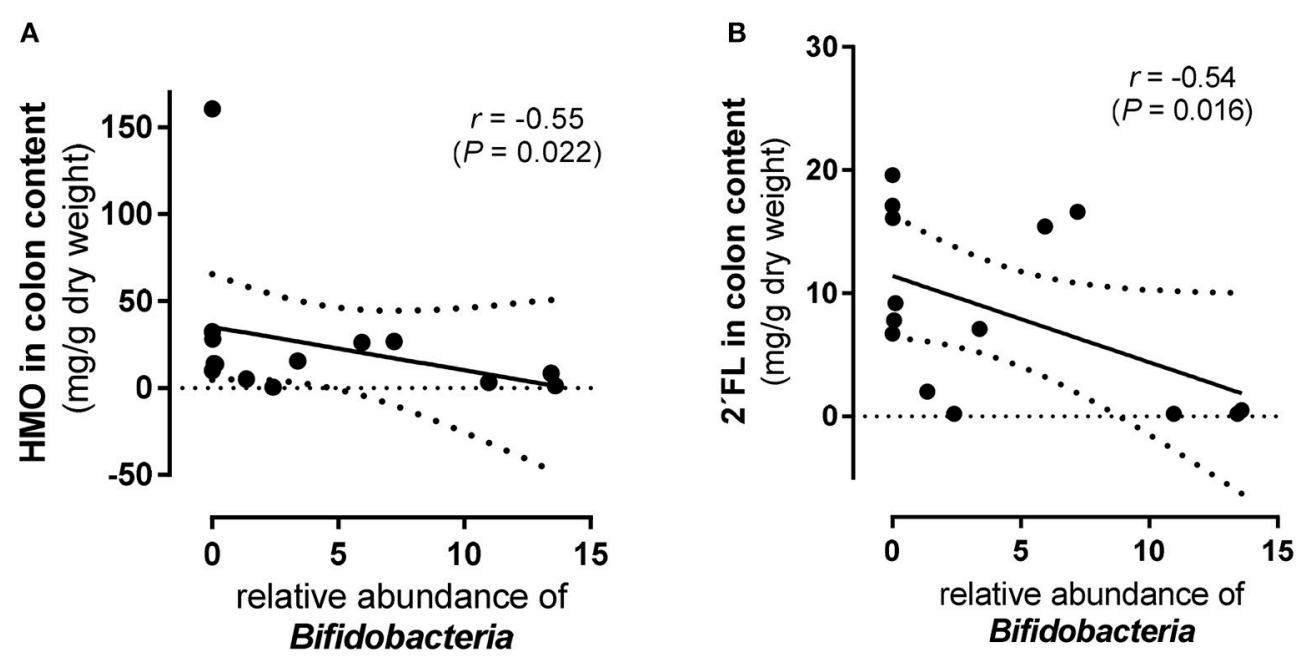

FIGURE 6 | Scatter plots of total and individual human milk oligosaccharides (HMO) and Bifidobacteria in colon content from preterm pigs fed the 4-HMO blend for 11 days (Experiment 3). Correlation of Bifidobacteria abundance with (A) total HMO level in colon content and (B) with $2^{\prime}$ fucosyllactose (FL). Spearman's coefficients $(r)$ and $p$-values $(P)$ are shown.

TABLE 3 | Correlation of total HMOs in colon and urine with NEC and intestinal permeability, respectively.

\begin{tabular}{|c|c|c|c|}
\hline No. & $\begin{array}{c}\text { Experiment } 1 \\
\text { (5 days, } 4-\mathrm{HMO}) \\
\text { [piglets, } n]\end{array}$ & $\begin{array}{c}\text { Experiment } 2 \\
\text { (5 days, 25-HMO) } \\
\text { [piglets, } n \text { ] }\end{array}$ & $\begin{array}{c}\text { Experiment } 3 \\
\text { (11 days, 4HMO) } \\
\text { [piglets, } n \text { ] }\end{array}$ \\
\hline \multicolumn{4}{|l|}{ NEC $^{a}$} \\
\hline All piglets & $-0.318^{\mathrm{nsc}}[17]$ & $-0.722^{\mathrm{ns}}[18]$ & $-0.316^{\text {ns }}[16]$ \\
\hline NEC & $-0.866^{\mathrm{ns}}[4]$ & $-0.764^{\star *} e[13]$ & $-0.476^{\mathrm{ns}}[9]$ \\
\hline No NEC & $-0.241^{\mathrm{ns}}[13]$ & $-0.001^{\mathrm{ns}}[5]$ & $-0.722^{\text {ns }}[7]$ \\
\hline \multicolumn{4}{|l|}{ PER $^{b}$} \\
\hline All piglets & $0.121^{\mathrm{ns}}[8]$ & $-0.318^{\mathrm{ns}}[11]$ & $0.400^{\text {ns }}[4]$ \\
\hline NEC & n.d. ${ }^{d}[2]$ & $-0.866^{\mathrm{ns}}[3]$ & n.d. [2] \\
\hline No NEC & $-0.116^{\mathrm{ns}}[6]$ & $-0.241^{\mathrm{ns}}[8]$ & n.d. [2] \\
\hline
\end{tabular}

a Cumulative NEC score: a score of $\geq 3$ in any of the evaluated regions (stomach, proximal, middle, distal small intestine or colon) was defined as NEC.

${ }^{b}$ Permeability: lactulose and mannitol contents were analyzed in urine and lactulose to mannitol concentration ratio was used as an indicator of intestinal permeability.

${ }^{c}$ Significance was given at ${ }^{* *} P<0.05$ (ns = not significant).

${ }^{d}$ n.d. = not determined. The limited sample size hampered detail statistical evaluation for subgroups.

e Significant correlation for $2^{\prime} F L(r=-0.67 ; P=0.01)$, difucosyllactose $(D F-L)(r=-0.69$; $P=0.008)$, LNFP $/ /(r=-0.72 ; P=0.0039), L N T(r=-0.65 ; P=0.0076), L N n T(r=$ $-0.59 ; P=0.0011)$ and disialyl-LNT (DSLNT) ( $r=-0.64 ; P=0.03)$, but not for 3-FL, LNFP I, LNFP III, sialyl-LNT (LST) a, LST b, 3'SL and 6'SL.

isomers (34). While 3-FL might be primarily utilized by gut microbiota, as it is low in both, colon content and in urine, LNFP I seems to selectively be taken up and excreted in relatively large amounts in urine (Figure 3B). Another interesting observation regarding $2^{\prime} \mathrm{FL}$ is that whenever the fecal excretion of $2^{\prime} \mathrm{FL}$ was high, the excretion of all other HMOs in the same animal was high as well suggesting an overall low microbial activity.

Regarding disease prevention, the acidic HMO, DSLNT, had remarkable NEC-preventing effects in newborn rats (19). In our preterm pigs, DSLNT was neither clearly related to NEC nor to bacterial colonization despite the high supplementation (about 7\% of the 25-HMO blend). These differences could reflect the immaturity of the gut and its bacterial colonization in preterm pigs, a prevailing dysbiosis, which is rather common in cesarean born preterm pigs, with unknown severity, or speciesspecific differences.

Large individual variability in fecal excretion of HMO have also been found for term and preterm infants fed human milk $(26,35,36)$. A gradual change in the fecal oligosaccharide profile in breastfed infants during the first six months postpartum has been reported, without LNT being identified in fecal samples (37). This is in contrast to our previous data $(26,35,36$, 38), where we detected LNT in all fecal samples from human term-born infants whenever HMO were excreted. In infants, there is a large variation in the amount and pattern of HMO excretions, ranging from large amounts to no excretion at all. The reason for this divergence is not known but may be related to a different gut microbial composition and, hence, a different HMO metabolism in the intestinal lumen. For example, LNT, the major core structure of HMO, was initially considered to be a unique growth promoter of Bifidobacterium longum subsp. infantis although others, e.g., Bifidobacteria breve also use intact LNT $(39,40)$. In future studies, it is important to relate HMO metabolism in the gut lumen to subspecies level. In the present study we observed that microbial colonization in the two 5-day experiments was limited to Firmicutes (Experiment 1) and relatively low numbers of Actinobacteria and Bacteriodetes (Experiment 2), consisting mainly of Clostridium (cluster I) and Enterococcus. In Experiment 3 (11-day supplementation of the 4-HMO blend), more bacterial phyla were found, with Proteobacteria (mainly Campylobacter and Helicobacter) as the predominant phylum. Bifidobacteria were also detected but in lower abundance. There were no changes in the microbial colonization of the pigs when supplementing formula with the 4-HMO blend. The high abundance of Proteobacteria in the 
11-day experiment is in agreement with observations in a study with preterm infants who were fed formula supplemented with $\mathrm{HMO}$ from pooled human milk (no specification of HMO were given) (41). The authors report low levels of Bifidobacteria and no Lactobacilli, concomitantly with increasing numbers of Clostridia and an unexpected trend toward an increase in Proteobacteria in both groups. Although the interpretation of the data from our piglets is difficult due to the large individual variation, the presence of Bifidobacteria, although low in total numbers, was correlated with total HMO contents, and specifically with $2^{\prime} \mathrm{FL}$ levels in colonic content. The importance of $2^{\prime} \mathrm{FL}$ particularly for Bifidobacteria through the interaction with a newly identified $\mathrm{ABC}$ transporter as a key genetic factor for the utilization of $2^{\prime} \mathrm{FL}$ and other fucosylated oligosaccharides has been thoroughly discussed by Matsuki et al. (42). This could be important, as recent reports indicated a strong association of the secretor genotype with the composition of Bifidobacteria in the human intestine $(43,44)$.

In conclusion, we found that (i) all pigs receiving HMO containing diets excreted these HMO via the colon and urine, but individual variations were large. This data resemble the situation in human infants with no clear excretion pattern neither in term nor in preterm infants; (ii) HMO supplemenation was not related to NEC, bacterial colonization or intestinal permeability; (iii) the 5-day supplementation of the complex 25-HMO blend led to low concentrations of 3-FL and LNFP I in colonic contents, indicating a preferred utilization of these two HMO; (iv) In colon, only the abundance of Bifidobacteria, although low in total numbers compared to other microorganisms, correlated with total HMO in colon content and specifically with $2^{\prime} \mathrm{FL}$, (v) Increasing the HMO supplementation period to 11 days lowered

\section{REFERENCES}

1. Bode L. Human milk oligosaccharides: every baby needs a sugar mama. Glycobiology. (2012) 22:1147-62. doi: 10.1093/glycob/cws074

2. Hennet T, Borsig L. Breastfed at Tiffany's. Trends Biochem Sci. (2016) 41:50818. doi: 10.1016/j.tibs.2016.02.008

3. Vazquez E, Barranco A, Ramirez M, Gruart A, Delgado-Garcia JM, MartinezLara E, et al. Effects of a human milk oligosaccharide, 2'-fucosyllactose, on hippocampal long-term potentiation and learning capabilities in rodents. J Nutr Biochem. (2015) 26:455-65. doi: 10.1016/j.jnutbio.2014. 11.016

4. McGuire M. Prebiotics and Probiotics in Human Milk: Origins and Functions of Milk-Borne Oligosaccharides and Bacteria. Saint Louis, MO: Elsevier Science (2016). p. 1508

5. Donovan SM, Comstock SS. Human milk oligosaccharides influence neonatal mucosal and systemic immunity. Ann Nutr Metab. (2016) 69(Suppl. 2):42-51. doi: $10.1159 / 000452818$

6. Urashima T, Hirabayashi J, Sato S, Kobata A. Human milk oligosaccharides as essential tools for basic and application studies on galectins. Trends Glycosci Glycotechnol. (2018) 30:SE51-65. doi: 10.4052/tigg.1734.1SE

7. Morozov V, Hansman G, Hanisch F-G, Schroten H, Kunz C. Human milk oligosaccharides as promising antivirals. Mol Nutr Food Res. (2018) 62:e1700679. doi: 10.1002/mnfr.201700679

8. Marriage BJ, Buck RH, Goehring KC, Oliver JS, Williams JA. Infants fed a lower calorie formula with 2'FL show growth and 2'FL uptake like breast-fed infants. J Pediatr Gastroenterol Nutr. (2015) 61:649-58. doi: 10.1097/MPG.0000000000000889 the fecal excretion of HMO. Thus, intestinal immaturity, together with delayed bacterial colonization and low bacterial diversity, may lead to a different metabolic fate of HMO during the first 1-2 weeks after preterm birth. A sufficient gastrointestinal maturation may be required to observe clear benefits of HMO, both locally in the gut and beyond.

\section{AUTHOR CONTRIBUTIONS}

The authors' responsibilities were as follows: SR, NS, PS, and SB designed research; SR, SO, and MR conducted research; SR, SK, and MR analyzed the data; SR, SK, PS, and SB wrote the paper; SR, SO, SK, CK, PS, and SB revised the manuscript. SR, PS, and SB had primary responsibility for the final content. All authors read and approved the final manuscript.

\section{FUNDING}

The study was supported by the Innovation Fund Denmark (NEOMUNE grant number 0603-00774B) and Glycom A/S (Lynby, Denmark). The funder Glycom A/S (Lynby, Denmark) provided the HMO blends for the study, but played no role in the study design, the collection, analysis or interpretation of data, the writing of this paper or the decision to submit it for publication.

\section{ACKNOWLEDGMENTS}

We thank Dr. Christian Borsch, Cordula Becker, and Christina Meyer from the University of Giessen for their great technical assistance.

9. Puccio G, Alliet P, Cajozzo C, Janssens E, Corsello G, Sprenger N, et al. Effects of infant formula with human milk oligosaccharides on growth and morbidity: a randomized multicenter trial. J Pediatr Gastroenterol Nutr. (2017) 64:624-31. doi: 10.1097/MPG.0000000000001520

10. Goehring KC, Kennedy AD, Prieto PA, Buck RH. Direct evidence for the presence of human milk oligosaccharides in the circulation of breastfed infants. PLoS ONE. (2014) 9:e101692. doi: 10.1371/journal.pone.0101692

11. Ruhaak LR, Stroble C, Underwood MA, Lebrilla CB. Detection of milk oligosaccharides in plasma of infants. Anal Bioanal Chem. (2014) 406:577584. doi: 10.1007/s00216-014-8025-z

12. Jantscher-Krenn E, Marx C, Bode L. Human milk oligosaccharides are differentially metabolised in neonatal rats. Br J Nutr. (2013) 110:640-50. doi: 10.1017/S0007114512005727

13. Vazquez E, Santos-Fandila A, Buck R, Rueda R, Ramirez M. Major human milk oligosaccharides are absorbed into the systemic circulation after oral administration in rats. Br J Nutr. (2017) 117:237-47. doi: $10.1017 /$ S0007114516004554

14. Hansen CF, Thymann T, Andersen AD, Holst JJ, Hartmann B, Hilsted L, et al. Rapid gut growth but persistent delay in digestive function in the postnatal period of preterm pigs. Am J Physiol Gastrointest Liver Physiol. (2016) 310:G550-60. doi: 10.1152/ajpgi.00221.2015

15. Sangild PT, Thymann T, Schmidt M, Stoll B, Burrin DG, Buddington RK. Invited review: the preterm pig as a model in pediatric gastroenterology. $J$ Anim Sci. (2013) 91:4713-29. doi: 10.2527/jas.2013-6359

16. Carlisle EM, Morowitz MJ. The intestinal microbiome and necrotizing enterocolitis. Curr Opin Pediatr. (2013) 25:382-7. doi: 10.1097/MOP.0b013e3283600e91 
17. Li M, Monaco MH, Wang M, Comstock SS, Kuhlenschmidt TB, Fahey GC, et al. Human milk oligosaccharides shorten rotavirus-induced diarrhea and modulate piglet mucosal immunity and colonic microbiota. ISME J. (2014) 8:1609-20. doi: 10.1038/ismej.2014.10

18. Neu J. Preterm infant nutrition, gut bacteria, and necrotizing enterocolitis. Curr Opin Clin Nutr Metab Care. (2015) 18:285-8. doi: 10.1097/MCO.0000000000000169

19. Jantscher-Krenn E, Zherebtsov M, Nissan C, Goth K, Guner YS, Naidu N, et al. The human milk oligosaccharide disialyllacto-N-tetraose prevents necrotising enterocolitis in neonatal rats. Gut. (2012) 61:1417-25. doi: 10.1136/gutjnl-2011-301404

20. Good M, Sodhi CP, Yamaguchi Y, Jia H, Lu P, Fulton WB, et al. The human milk oligosaccharide 2'-fucosyllactose attenuates the severity of experimental necrotising enterocolitis by enhancing mesenteric perfusion in the neonatal intestine. $\mathrm{Br} J$ Nutr. (2016) 116:1175-87. doi: 10.1017/S0007114516 002944

21. Autran CA, Schoterman MH, Jantscher-Krenn E, Kamerling JP, Bode L. Sialylated galacto-oligosaccharides and 2'-fucosyllactose reduce necrotising enterocolitis in neonatal rats. Br J Nutr. (2016) 116:294-9. doi: 10.1017/S0007114516002038

22. Wu RY, Li B, Koike $Y$, Määttänen $\mathrm{P}$, Miyake $\mathrm{H}$, Cadete $\mathrm{M}$, et al. Human milk oligosaccharides increase mucin expression in experimental necrotizing enterocolitis. Mol Nutr Food Res. (2018) 2018:e1800658. doi: $10.1002 / \mathrm{mnfr} .201800658$

23. Rasmussen SO, Martin L, Ostergaard MV, Rudloff S, Roggenbuck M, Nguyen $\mathrm{DN}$, et al. Human milk oligosaccharide effects on intestinal function and inflammation after preterm birth in pigs. J Nutr Biochem. (2017) 40:141-54. doi: 10.1016/j.jnutbio.2016.10.011

24. Thymann T, Burrin DG, Tappenden KA, Bjornvad CR, Jensen SK, Sangild PT. Formula-feeding reduces lactose digestive capacity in neonatal pigs. Br J Nutr. (2006) 95:1075-81. doi: 10.1053/j.gastro.2006. 02.026

25. Kunz C, Meyer C, Collado MC, Geiger L, García-Mantrana I, Bertua-Ríos B, et al. Influence of gestational age, secretor, and lewis blood group status on the oligosaccharide content of human milk. J Pediatr Gastroenterol Nutr. (2017) 64:789-98. doi: 10.1097/MPG.0000000000001402

26. Dotz V, Rudloff S, Meyer C, Lochnit G, Kunz C. Metabolic fate of neutral human milk oligosaccharides in exclusively breast-fed infants. Mol Nutr Food Res. (2015) 59:355-64. doi: 10.1002/mnfr.201400160

27. Rasmussen SO, Martin L, Ostergaard MV, Rudloff S, Li Y, Roggenbuck $\mathrm{M}$, et al. Bovine colostrum improves neonatal growth, digestive function, and gut immunity relative to donor human milk and infant formula in preterm pigs. Am J Physiol Gastrointest Liver Physiol. (2016) 311:G480-91. doi: 10.1152/ajpgi.00139.2016

28. Neefs JM, de Wachter R. A proposal for the secondary structure of a variable area of eukaryotic small ribosomal subunit RNA involving the existence of a pseudoknot. Nucleic Acids Res. (1990) 18:5695-704.

29. Yu Y, Lee C, Kim J, Hwang S. Group-specific primer and probe sets to detect methanogenic communities using quantitative real-time polymerase chain reaction. Biotechnol Bioeng. (2005) 89:670-9. doi: 10.1002/bit.20347

30. Sundberg C, Al-Soud WA, Larsson M, Alm E, Yekta SS, Svensson BH, et al. 454 pyrosequencing analyses of bacterial and archaeal richness in 21 full-scale biogas digesters. FEMS Microbiol Ecol. (2013) 85:612-26. doi: 10.1111/1574-6941.12148

31. Schloss PD, Westcott SL, Ryabin T, Hall JR, Hartmann M, Hollister $\mathrm{EB}$, et al. Introducing mothur: open-source, platform-independent, community-supported software for describing and comparing microbial communities. Appl Environ Microbiol. (2009) 75:7537-41. doi: 10.1128/AEM. 01541-09
32. Edgar RC. UPARSE: highly accurate OTU sequences from microbial amplicon reads. Nat Methods. (2013) 10:996-8. doi: 10.1038/nmeth.2604

33. Paulson JN, Stine OC, Bravo HC, Pop M. Differential abundance analysis for microbial marker-gene surveys. Nat Methods. (2013) 10:1200-2. doi: $10.1038 /$ nmeth. 2658

34. Weichert S, Koromyslova A, Singh BK, Hansman S, Jennewein S, Schroten H, et al. Structural basis for norovirus inhibition by human milk oligosaccharides. J Virol. (2016) 90:4843-8. doi: 10.1128/JVI.03223-15

35. Rudloff S, Obermeier S, Borsch C, Pohlentz G, Hartmann R, Brosicke H, et al. Incorporation of orally applied (13)C-galactose into milk lactose and oligosaccharides. Glycobiology. (2006) 16:477-87. doi: 10.1093/glycob/cwj092

36. Rudloff S, Kunz C. Milk oligosaccharides and metabolism in infants. Adv Nutr. (2012) 3:398. doi: 10.3945/an.111.001594

37. Albrecht S, Schols HA, van den Heuvel, Ellen GHM, Voragen AG, Gruppen $\mathrm{H}$. Occurrence of oligosaccharides in feces of breast-fed babies in their first six months of life and the corresponding breast milk. Carbohydr Res. (2011) 346:2540-50. doi: 10.1016/j.carres.2011.08.009

38. Dotz V, Rudloff S, Blank D, Lochnit G, Geyer R, Kunz C. 13C-labeled oligosaccharides in breastfed infants' urine: individual-, structure- and timedependent differences in the excretion. Glycobiology. (2014) 24:185-94. doi: 10.1093/glycob/cwt099

39. Garrido D, Ruiz-Moyano S, Mills DA. Release and utilization of $\mathrm{N}$-acetyl-D-glucosamine from human milk oligosaccharides by Bifidobacterium longum subsp. infantis. Anaerobe. (2012) 18:430-5. doi: 10.1016/j.anaerobe.2012.04.012

40. Asakuma S, Hatakeyama E, Urashima T, Yoshida E, Katayama T, Yamamoto $\mathrm{K}$, et al. Physiology of consumption of human milk oligosaccharides by infant gut-associated bifidobacteria. J Biol Chem. (2011) 286:34583-92. doi: $10.1074 /$ jbc.M111.248138

41. Underwood MA, Arriola J, Gerber CW, Kaveti A, Kalanetra KM, Kananurak A, et al. Bifidobacterium longum subsp. infantis in experimental necrotizing enterocolitis: alterations in inflammation, innate immune response, and the microbiota. Pediatr Res. (2014) 76:326-33. doi: 10.1038/pr.2014.102

42. Matsuki T, Yahagi K, Mori H, Matsumoto H, Hara T, Tajima S, et al. A key genetic factor for fucosyllactose utilization affects infant gut microbiota development. Nat Commun. (2016) 7:11939. doi: 10.1038/ncomms11939

43. Lewis ZT, Totten SM, Smilowitz JT, Popovic M, Parker E, Lemay DG, et al. Maternal fucosyltransferase 2 status affects the gut bifidobacterial communities of breastfed infants. Microbiome. (2015) 3:13. doi: 10.1186/s40168-015-0071-z

44. Wacklin P, Makivuokko H, Alakulppi N, Nikkila J, Tenkanen H, Rabina J, et al. Secretor genotype (FUT2 gene) is strongly associated with the composition of Bifidobacteria in the human intestine. PLOS ONE. (2011) 6:e20113. doi: 10.1371/journal.pone.0020113

Conflict of Interest Statement: NS is employed by Nestec S.A. at the Nestle Research Center.

The remaining authors declare that the research was conducted in the absence of any commercial or financial relationships that could be construed as a potential conflict of interest.

Copyright (C) 2019 Rudloff, Kuntz, Ostenfeldt Rasmussen, Roggenbuck, Sprenger, Kunz, Sangild and Brandt Bering. This is an open-access article distributed under the terms of the Creative Commons Attribution License (CC BY). The use, distribution or reproduction in other forums is permitted, provided the original author(s) and the copyright owner(s) are credited and that the original publication in this journal is cited, in accordance with accepted academic practice. No use, distribution or reproduction is permitted which does not comply with these terms. 